\title{
Structural basis for the oncogenic signalling complex formed by Grb2 and Gab2 in Her2 (ErbB2/Neu)-driven breast cancers and CML cells
}

\author{
T Tsirka*1, M Harkiolaki², M Lewitzky¹, H Wecklein ${ }^{3}$, L Bird ${ }^{4}$, EY Jones², \\ T Raabe ${ }^{3}$, N OReilly ${ }^{5}$ and SM Feller ${ }^{1}$
}

\begin{abstract}
Address: ${ }^{1}$ Weatherall Insitute of Molecular Medicine, Cell Signalling Group, University of Oxford, Oxford, UK, ${ }^{2}$ Division of Structural Biology, Henry Wellcome Building of Genomic Medicine, Oxford, UK, ${ }^{3}$ Institute of Medical Radiation and Cell Research, University of Würzburg, Würzburg, Germany, ${ }^{4}$ Oxford Module Consortium, OPPF, Wellcome Trust Centre, Oxford, UK and ${ }^{5}$ CRUK Protein and Peptide Chemistry Laboratory, London Research Institute, London, UK

* Corresponding author
\end{abstract}

from 12th Joint Meeting of the Signal Transduction Society (STS). Signal Transduction: Receptors, Mediators and Genes

Weimar, Germany. 29-31 October 2008

Published: 26 February 2009

Cell Communication and Signaling 2009, 7(SuppI I):A39 doi:10.1186/I478-8IIX-7-SI-A39

This abstract is available from: http://www.biosignaling.com/content/7/SI/A39

(c) 2009 Tsirka et al; licensee BioMed Central Ltd.

The adaptor protein Grb2 and the large multi-site docking protein Gab2 form a complex that is crucial for the oncogenic signalling of some cancer cells. Binding of the C-terminal SH3 (SH3C) domain of Grb2 to Gab2 is essential for the interaction, but molecular details of the complex formation have remained undefined. Using peptide array SH3 overlay blots, isothermal titration calorimetry and protein crystallography, two atypical SH3 domain binding sites in Gab2 (Gab2-1 and Gab2-2) were confirmed and characterised. Gab2-1 has considerable similarity to an epitope in the cell cycle regulator p27Kip1 that also binds Grb2SH3C. The SH3C binding sites in Gab2 differ approximately 5 -fold in their affinity and also with respect to the residues important for the interaction apart from a shared core motif RxxK. Both SH3 domain interaction sites in Gab2 are conserved throughout evolution and found, for example, in the Gab homolog daughter of sevenless (Dos) of Drosophila melanogaster, which binds to the Grb2 homolog Drk. Mutagenesis of the Drk binding sites in Dos in vivo impairs fly eye and wing vein development and documents that both sites are functionally important. A high-resolution crystal structure was generated of the Gab2-2 epitope in complex with the Grb2SH3C domain. This reveals a binding mode reminiscent of the interaction of haematopoietic Grb2-relative Mona/Gads with the Tcell adaptor SLP-76 and haematopoietic progenitor kinase
1 (HPK1). However, subtle differences exist, which could potentially be exploited to generate SH3C domain inhibitors with preference for a specific Grb2 family member. 